\title{
密度泛函理论研究 5-单苯基卟啉分子的几何结构和拉曼光谱"
}

\author{
章应辉阮文娟吴扬 \\ (南开大学化学系, 天津 300071)
}

\begin{abstract}
摘要 利用密度泛函理论(DFT)计算了 5 -单苯基吓啉 $\left(\mathrm{H}_{2} \mathrm{MPP}\right)$ 的几何结构和拉曼振动频率. 计算表明, 单个次甲 基位置上的苯基取代降低了卟啉骨架大环的对称性. 苯基团取代对次甲基位置附近结构的影响较大, 而对吡咯 环结构的影响较小. 计算给出的拉曼振动频率(校正因子为 0.971 )与实验测量数据吻合较好, 均方根误差(RMS) 小于 $6.7 \mathrm{~cm}^{-1}$. 根据理论计算结果对实测拉曼光谱进行了指认, 计算分析和实验观察同时表明, 单个次甲基位置 上的苯基取代导致卟啉大环的一些平面内简正振动, 如 $\nu_{6} 、 \nu_{20}, \nu_{24}$ 和 $\nu_{32}$ 等简正振动发生分裂. 分析认为其根本原 因为单苯基取代导致的叶啉骨架大环对称性的降低.
\end{abstract}

关键词：密度泛函理论，5-苯基卟啉，拉曼光谱

中图分类号： O641， O657.37

利用拉曼光谱研究卟啉类分子的结构一直是人 们关注的一个重点 ${ }^{[-2]}$. 实验上可以通过同位素取代 方法来研究卟啉的拉曼振动结构 ${ }^{[3-4]}$; 理论计算方面, 密度泛函理论(DFT)得到了广泛的应用. 对于忽略非 谐性振动效应导致的振动频率计算值的偏高, 可以 通过两种方法进行校正, 一种是校准量子力学法 $(\mathrm{SQM})^{[5-6]}$, 即对各种类型键的计算力常数逐一进行 校正; 另一种较为简单的方法是用同一个校正因子 对所有计算频率进行校正 ${ }^{[-8]}$, 也可以得到比较满意 的结果.

目前的理论研究主要集中于卟吩 $\left(\mathrm{H}_{2} \mathrm{P}\right)^{[6]}$ 和 $m e s o$-四苯基卟啉分子 $\left(\mathrm{H}_{2} \mathrm{TPP}\right)^{[r]}$ 等对称性较高的叶 啉分子. 而不对称取代卟啉分子的研究相对较少. 在本文中, 我们利用 DFT 研究了 5-单苯基卟啉 $\left(\mathrm{H}_{2} \mathrm{MPP}\right.$, 结构见图 1$)$ 的几何结构和拉曼振动频率, 利 用校正因子 (0.971) 对所有计算频率进行了校正. 所 得结果与实验测量频率吻合较好. 理论计算分析表 明, 次甲基上的单苯基取代对次甲基桥附近结构影 响较大, 而对吡咯环结构的影响相对较小. 同时, 单 个次甲基上的苯基取代还导致卟啉大环平面结构对 称性的降低, 这导致了一些卟啉大环平面内简正振 动发生分裂.

\section{1 实验部分和理论计算步骤}

$\mathrm{H}_{2} \mathrm{MPP}$ 的合成按文献[9]进行. 共振拉曼光谱 (RRS) 在 Renishaw Invia 显微拉曼光谱仪上测得, 使 用 $\mathrm{Ar}^{\mathrm{N}}$ 激光器的 $514.5 \mathrm{~nm}$ 线为激发光 $(1 \mathrm{~mW})$, 利用 背散射方式收集散射光. 非共振拉曼光谱(NRS)在 Bruker RFS100/S FT-Raman 光谱仪上测得. 利用 $\mathrm{CW} N \mathrm{~N}: \mathrm{YAG}$ 激光器的 $1064 \mathrm{~nm}$ 线做激发光, 样品 在 $15 \mathrm{~mW}$ 的激光照射下连续扫描 1000 次. 所有拉 曼光谱的分辨率均优于 $2 \mathrm{~cm}^{-1}$.

密度泛函理论计算使用 Gaussian 03 软件 ${ }^{[10]}$ 进

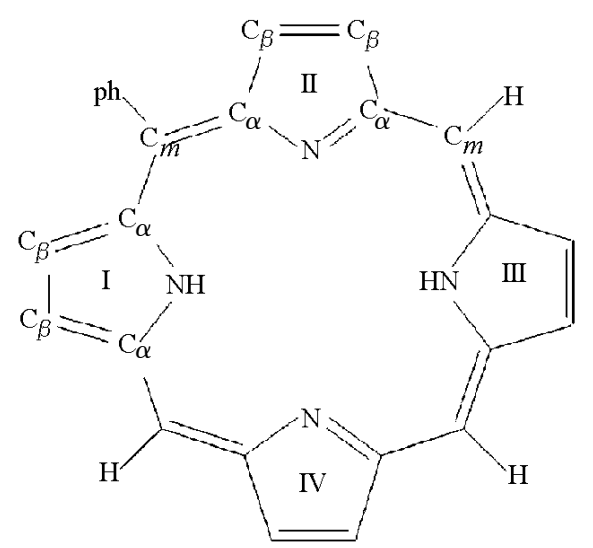

图 $1 \mathrm{H}_{2} \mathrm{MPP}$ 的结构图

Fig.1 Labeling diagram of $\mathrm{H}_{2} \mathrm{MPP}$

2005-05-16 收到初稿, 2005-07-29 收到修改稿. 联系人:章应辉(E-mail: zhangyhi@ nankai.edu.cn; Tel:022-81177012). *国家自然科学基 金(20303009,20271030)资助项目 
行, $\mathrm{H}_{2} \mathrm{MPP}$ 初始结构参照已知的 $\mathrm{H}_{2} \mathrm{P}$ 结构构建 ${ }^{[5]}$. 将一个次甲基位置上的 $\mathrm{H}$ 原子用苯基团代替, 即得 到 $\mathrm{H}_{2} \mathrm{MPP}$ 的初始结构. 初始结构优化是在没有对称 性限制下利用 B3LYP 泛函 ${ }^{[11]}$ 和 6-31G 基组 ${ }^{[12]}$ 进 行. 将所得结果在 6-31G $(d)$ 基组和适当的对称性限 制下进一步优化, 得到最终结构. 在此结构基础上进 行的频率计算没有发现虚频的存在, 表明最终的优 化结构 ( $C_{1}$ 点群)是稳定的. 将计算所得振动频率乘 以校正因子 0.971 , 得到最终计算频率. 通过与实测 拉曼光谱数据的比较, 表明计算频率的均方根误差 小于 $6.7 \mathrm{~cm}^{-1}$.

\section{2 结果和讨论}

\section{$2.1 \mathrm{H}_{2} \mathrm{MPP}$ 基态几何结构}

$\mathrm{H}_{2} \mathrm{MPP}$ 和 $\mathrm{H}_{2} \mathrm{P}$ 的主要计算结构参数列于表 1 中. 计算结果表明, $\mathrm{H}_{2} \mathrm{MPP}$ 和 $\mathrm{H}_{2} \mathrm{P}$ 的稳定结构分别 为 $C_{1}$ 和 $D_{2 h}$ 点群. $\mathrm{H}_{2} \mathrm{MPP}$ 分子对称性的降低首先可 归因于单个次甲基桥位上的苯基取代导致的整个卟 啉分子对称性的降低(见图 1). 计算同时表明, 苯基 团和吓啉大环间的二面角约为 $64^{\circ}$, 这进一步降低 了整个卟啉分子的对称性. 此外, 单苯基取代还导致

表 1 DFT 计算的 $\mathrm{H}_{2} M P P$ 和 $\mathrm{H}_{2} \mathrm{P}$ 的部分结构参数

Table 1 Selected calculated structural parameters of $\mathrm{H}_{2} \mathrm{MPP}$ and $\mathrm{H}_{2} \mathrm{P}$ by DFT

\begin{tabular}{|c|c|c|}
\hline & $\mathrm{H}_{2} \mathrm{P}\left(D_{2 h}\right)$ & $\mathrm{H}_{2} \mathrm{MPP}^{\mathrm{a}}\left(C_{1}\right)$ \\
\hline \multicolumn{3}{|c|}{ Bond length (nm) } \\
\hline \multirow{2}{*}{$\begin{array}{ccc}\mathrm{C}_{\beta} \mathrm{C}_{\beta} & \mathrm{pp} \\
& \mathrm{d}_{1}\end{array}$} & 0.1372 & 0.1372 \\
\hline & 0.1356 & 0.1356 \\
\hline \multirow{2}{*}{$\mathrm{C}_{\alpha} \mathrm{C}_{\beta}$} & 0.1435 & 0.1435 \\
\hline & 0.1460 & 0.1460 \\
\hline \multirow{2}{*}{$\begin{array}{ll}\mathrm{C}_{\alpha} \mathrm{N} & \mathrm{pp} \\
& \mathrm{d}_{\mathrm{p}}\end{array}$} & 0.1373 & 0.1373 \\
\hline & 0.1364 & 0.1364 \\
\hline $\mathrm{C}_{\alpha} \mathrm{C}_{m}$ & 0.1397 & 0.1400 \\
\hline \multicolumn{3}{|c|}{ Bond angle $\left(^{\circ}\right)$} \\
\hline $\mathrm{C}_{\alpha} \mathrm{C}_{\beta} \mathrm{C}_{\beta} \mathrm{pp}$ & 108.0 & 108.1 \\
\hline $\mathrm{dp}$ & 106.2 & 106.2 \\
\hline $\mathrm{NC}_{\alpha} \mathrm{C}_{\beta} \mathrm{pp}$ & 106.5 & 106.5 \\
\hline $\mathrm{dp}$ & 111.1 & 111.1 \\
\hline $\mathrm{C}_{\alpha} \mathrm{NC}_{\alpha} \mathrm{pp}$ & 110.9 & 110.8 \\
\hline $\mathrm{dp}$ & 105.4 & 105.5 \\
\hline $\mathrm{C}_{\alpha} \mathrm{C}_{m} \mathrm{C}_{\alpha}$ & 127.1 & 126.7 \\
\hline \multicolumn{3}{|c|}{ Dihedral angle $\left(^{\circ}\right)$} \\
\hline $\mathrm{NC}_{\alpha} \mathrm{C}_{m} \mathrm{C}_{\alpha}$ & 0.00 & 0.61 \\
\hline
\end{tabular}

${ }^{a}$ averaged value; ${ }^{b}$ pp: protonated pyrrole ring; 'dp: deprotonated pyrrole ring
吓啉骨架大环对称性的降低. 对于 $\mathrm{H}_{2} \mathrm{P}$ 和 $\mathrm{H}_{2} \mathrm{TPP}$ 等 吓啉分子, 四个次甲基桥位置的结构是相同的, 其四 个吡咯环可以分为质子化和非质子化吡咯两类, 不 同类吡咯环结构参数不同, 但同类吡咯环的结构参 数相同 (见表 1). 而在 $\mathrm{H}_{2} \mathrm{MPP}$ 分子中, 四个吡咯环结 构参数互不相同, 同时四个次甲基桥位置的结构也 有差异.

计算表明苯基取代主要对卟啉环中 $\mathrm{C}_{m}$ 原子附 近结构有较大影响, 单个苯基团的引人导致 $\mathrm{H}_{2} \mathrm{MPP}$ 分子的四个次甲基桥结构互不相同. 苯基团取代使 $\mathrm{C}_{m}$ 原子向远离吓啉环中心的方向移动, 导致该次甲 基位置附近 $\mathrm{C}_{\alpha} \mathrm{C}_{m}$ 键长的增大和 $\mathrm{C}_{\alpha} \mathrm{C}_{m} \mathrm{C}_{\alpha}$ 键角的减 小. 与 $\mathrm{H}_{2} \mathrm{P}$ 分子的理论计算结果相比较, 苯基团取代 的次甲基位置上, $\mathrm{C}_{\alpha} \mathrm{C}_{m}$ 键的平均键长由 $\mathrm{H}_{2} \mathrm{P}$ 的 $0.1397 \mathrm{~nm}$ 增加到 $0.1400 \mathrm{~nm}$, 而 $\mathrm{C}_{\alpha} \mathrm{C}_{m} \mathrm{C}_{\alpha}$ 键角则由 $\mathrm{H}_{2} \mathrm{P}$ 的 $127.1^{\circ}$ 下降到 $126.7^{\circ}$. 与苯基团取代的次甲 基相对称的次甲基的结构也有相同趋势的变化, 只 是幅度较小. 同时, 这两个次甲基间的 $\mathrm{C}_{m} \mathrm{C}_{m}$ 间距为 $0.7014 \mathrm{~nm}$, 明显大于 $\mathrm{H}_{2} \mathrm{P}$ 的 $0.6877 \mathrm{~nm}$. 另两个次甲 基位置上也发生了 $\mathrm{C}_{\alpha} \mathrm{C}_{m}$ 键长的增加和 $\mathrm{C}_{\alpha} \mathrm{C}_{m} \mathrm{C}_{\alpha}$ 键角 的减小, 但相应的 $\mathrm{C}_{m} \mathrm{C}_{m}$ 轴长 $(0.6767 \mathrm{~nm})$ 小于 $\mathrm{H}_{2} \mathrm{P}$ 的 $\mathrm{C}_{m} \mathrm{C}_{m}$ 轴长 $(0.6877 \mathrm{~nm})$. 总的来说, 与 $\mathrm{H}_{2} \mathrm{P}$ 分子相比, $\mathrm{H}_{2} \mathrm{MPP}$ 分子 $\mathrm{C}_{\alpha} \mathrm{C}_{m}$ 键长平均值和 $\mathrm{C}_{\alpha} \mathrm{C}_{m} \mathrm{C}_{\alpha}$ 键角平均 值分别增加了 $0.0003 \mathrm{~nm}$ 和减小了 $0.4^{\circ}$.

计算表明, 单苯基取代也导致吡咯环结构发生 不同程度的变化, 但变化幅度相对较小, 键长和键角 的变化幅度不超过 $0.0001 \mathrm{~nm}$ 和 $0.1^{\circ}$. 其中, 靠近苯 基取代的次甲基桥的吡咯环(环 I 和环 II)结构的变 化幅度要大于其他两个吡咯环(环III 和环IV)结构的 变化.

相对于平面内的结构变化, 单苯基取代导致的 卟啉大环的面外形变较小. $\mathrm{H}_{2} \mathrm{MPP}$ 的二面角 $\mathrm{NC}_{\alpha} \mathrm{C}_{m} \mathrm{C}_{\alpha}$ 的平均值只有 $0.61^{\circ}$, 可以认为 $\mathrm{H}_{2} \mathrm{MPP}$ 的 叶啉大环基本上保持平面结构.

\section{$2.2 \mathrm{H}_{2} \mathrm{MPP}$ 的拉曼振动分解}

我们主要考察 $\mathrm{H}_{2} \mathrm{MPP}$ 分子吓啉大环的骨架振 动. 将次甲基上的苯环简化为一个单原子基团, 这样 得到的卟啉分子结构模型 $\left(D_{2 h}\right.$ 点群)共有 38 个原子, 相应地有 $2 n-3=73(n=38)$ 个面内简正振动：

$$
\Gamma_{\text {in-plane }}=19 A_{\mathrm{g}}+18 B_{1 \mathrm{~g}}+18 B_{\mathrm{lu}}+18 B_{2 \mathrm{u}}
$$

$A_{\mathrm{g}}$ 和 $B_{\mathrm{lg}}$ 对称性的振动为吓啉环面内拉曼活性振动, $B_{1 \mathrm{u}}$ 和 $B_{2 \mathrm{u}}$ 为卟啉环面内红外活性振动. 在 $C_{1}$ 群的卟 啉分子中, 这些振动全部变为 $A$ 对称性的振动. 理 
论计算也显示, $\mathrm{H}_{2} \mathrm{MPP}$ 分子的所有简正振动都同时 具有拉曼活性和红外活性, 这应归因于 $\mathrm{H}_{2} \mathrm{MPP}$ 分子 中心对称性的消失. 但是计算也显示, 具有可观的理 论拉曼强度的仍然基本上是那些在 $D_{2 h}$ 点群下为拉 曼活性的简正振动, 尤其是面内简正振动(即 $A_{\mathrm{g}}$ 和 $B_{\mathrm{lg}}$ 振动). 这表明卟啉大环仍然具有相对较高的对 称性. 下面我们主要对拉曼光谱中有关吓啉大环骨 架的面内振动, 即 $A_{\mathrm{g}}$ 和 $B_{\mathrm{lg}}$ 振动进行分析指认.

\section{3 卟啉环平面内拉曼振动频率指认}

实测的共振拉曼光谱(RRS)和非共振拉曼光谱 (NRS) 见图 2 , 相应的拉曼振动频率数据列于表 2 中. 我们将计算得到的频率数据转化为 Lorentzian 峰形 的拉曼光谱(半峰宽为 $4 \mathrm{~cm}^{-1}$ ), 也显示在图 2 中. 为 了便于比较, 我们这里采用与文献[3,13]相同的简正 振动标记方法.

\subsection{1 $\mathrm{C}_{\alpha} \mathrm{C}_{m}$ 键伸缩振动}

不对称的 $\mathrm{C}_{\alpha} \mathrm{C}_{m}$ 键伸缩振动标记为 $\nu_{10}$ 和 $\nu_{19}$ 简 正振动, $\mathrm{H}_{2} \mathrm{MPP}$ 的 $\nu_{10}$ 振动的计算频率为 $1606 \mathrm{~cm}^{-1}$, 可以指认为实测拉曼光谱中的 $1605 \mathrm{~cm}^{-1}$ 带, 该带在 实测 NRS 谱中较弱, 但在实测 RRS 谱中较强, 这是 由于实测 RRS 谱所用的激发线 $(514.5 \mathrm{~nm})$ 靠近 $\mathrm{H}_{2} \mathrm{MPP}$ 的 $Q$ 电子吸收峰, 从而可使在 $D_{2 h}$ 点群下原 本为 $B_{1 \mathrm{~g}}$ 对称性的 $\nu_{10}$ 振动通过 Jahn-Teller 效应而 增加, 该振动的理论褪偏比值(0.649)也表明该振动 为褪偏的.

理论计算表明 $\nu_{19}$ 简正振动分裂为两个独立的

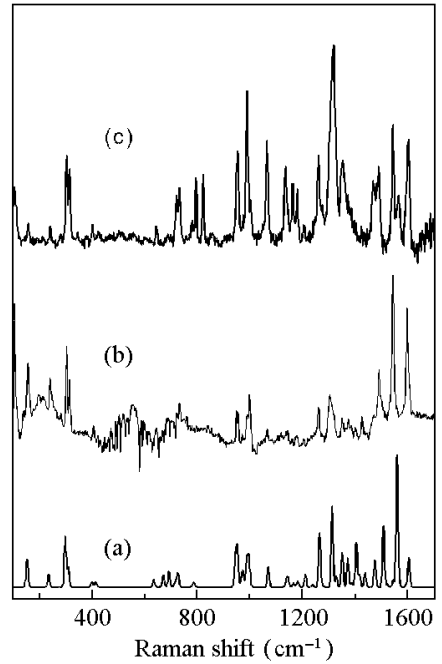

图 2 固体 $\mathrm{H}_{2} \mathrm{MPP}$ 拉曼光谱

\section{Fig.2 Raman spectra of solid $\mathrm{H}_{2} \mathrm{MPP}$}

(a) caculated Raman spectrum ( $F W H M=4 \mathrm{~cm}^{-1}$ ); (b) NRS (with $1064 \mathrm{~nm}$ excitation); (c) RRS (with $514.5 \mathrm{~nm}$ excitation)
简正振动, 分别主要包含吡咯环 I 和吡咯环III周围 的 $\mathrm{C}_{\alpha} \mathrm{C}_{m}$ 键的伸缩振动. 这两个振动的理论计算频率 分别为 1569 和 $1599 \mathrm{~cm}^{-1}$, 分别对应实测 RRS 拉曼 谱中的 1565 和 $1590 \mathrm{~cm}^{-1}$ 带. 我们认为单个苯基团 取代导致的四个次甲基桥位置结构的差异是 $\nu_{19}$ 简 正振动发生分裂的主要原因.

对称的 $\mathrm{C}_{\alpha} \mathrm{C}_{m}$ 键伸缩振动标记为 $\nu_{3}$ 和 $\nu_{28}$ 简正 振动, 其理论计算频率分别为 1439 和 $1476 \mathrm{~cm}^{-1}$, 分 别对应于实测光谱中的 1428 和 $1473 \mathrm{~cm}^{-1}$ 带.

\subsection{2 $\mathrm{C}_{\beta} \mathrm{C}_{\beta}$ 键伸缩振动}

质子化吡咯环的 $\mathrm{C}_{\beta} \mathrm{C}_{\beta}$ 键与非质子化吡咯环的

\section{表 $2 \mathrm{H}_{2}$ MPP 卟啉环的面内拉曼活性振动频率 $\left(\mathrm{cm}^{-1}\right)$}

Table 2 The calculated and measured in-plane Raman frequencies $\left(\mathrm{cm}^{-1}\right)$ of the porphyrin ring of $\mathrm{H}_{2} \mathrm{MPP}$

\begin{tabular}{|c|c|c|c|c|c|}
\hline \multirow{2}{*}{ mode } & \multirow{2}{*}{ Sym. $^{\text {a }}$} & \multicolumn{2}{|c|}{$f_{\mathrm{H}_{2} \mathrm{MPP}}$} & \multirow{2}{*}{$\Delta^{\mathrm{b}}$} & \multirow{2}{*}{ Assignment $^{\mathrm{c}}$} \\
\hline & & Cal. & Obs. & & \\
\hline$\nu_{10}$ & $A_{\mathrm{g}}$ & 1606 & 1605 & 1 & $\nu\left(\mathrm{C}_{\alpha} \mathrm{C}_{m}\right)$ \\
\hline$\nu_{19}$ & $B_{\mathrm{lg}}$ & $1599 / 1569$ & $1590 / 1565$ & $9 / 4$ & $\nu\left(\mathrm{C}_{\alpha} \mathrm{C}_{m}\right)_{\text {as }}$ \\
\hline$\nu_{2}$ & $A_{\mathrm{g}}$ & 1561 & 1544 & 17 & $\nu\left(\mathrm{C}_{\beta} \mathrm{C}_{\beta}\right)$ \\
\hline$\nu_{11}$ & $A_{\mathrm{g}}$ & 1509 & 1492 & 17 & $\nu\left(\mathrm{C}_{\beta} \mathrm{C}_{\beta}\right)$ \\
\hline$\nu_{28}$ & $B_{\lg }$ & 1476 & 1473 & 3 & $\nu\left(\mathrm{C}_{\alpha} \mathrm{C}_{m}\right)$ \\
\hline$\nu_{3}$ & $A_{\mathrm{g}}$ & 1439 & 1428 & 11 & $\nu\left(\mathrm{C}_{\alpha} \mathrm{C}_{m}\right)$ \\
\hline$\nu_{12}$ & $A_{\mathrm{g}}$ & 1406 & 1406 & 0 & $\nu(\text { pyr-half-ring })_{\mathrm{S}}$ \\
\hline$\nu_{20}$ & $B_{\mathrm{lg}}$ & $1388 / 1373$ & $1387 / 1378$ & $1 /-5$ & $\nu$ (pyr-quarter-ring $)$ \\
\hline$\nu_{4}$ & $A_{\mathrm{g}}$ & 1356 & 1354 & 2 & $\nu$ (pyr-half-ring) \\
\hline$\nu_{29}$ & $B_{\lg }$ & 1314 & 1307 & 7 & $\nu$ (pyr-quarter-ring) \\
\hline$\nu_{26}$ & $B_{\lg }$ & 1329 & 1328 & 1 & $\delta\left(\mathrm{C}_{\beta} \mathrm{H}\right)_{\mathrm{as}}$ \\
\hline$\nu_{1}$ & $A_{\mathrm{g}}$ & 1265 & 1264 & 1 & $v\left(\mathrm{C}_{m}-\mathrm{ph}\right)$ \\
\hline$\nu_{34}$ & $B_{\lg }$ & 1184 & 1182 & 2 & $\delta\left(\mathrm{C}_{\beta} \mathrm{H}\right)_{\mathrm{as}}$ \\
\hline$\nu_{17}$ & $A_{\mathrm{g}}$ & 1069 & 1065 & 4 & $\delta\left(\mathrm{C}_{\beta} \mathrm{H}\right)$ \\
\hline$\nu_{9}$ & $A_{\mathrm{g}}$ & 1071 & 1071 & 0 & $\delta\left(\mathrm{C}_{\beta} \mathrm{H}\right)$ \\
\hline$\nu_{30}$ & $B_{\lg }$ & 1002 & 1002 & 0 & $\nu$ (pyr-half-ring) \\
\hline$\nu_{15}$ & $A_{\mathrm{g}}$ & 989 & 992 & -3 & $\nu($ pyr-breathing $)$ \\
\hline$\nu_{22}$ & $B_{\lg }$ & 974 & 975 & -1 & $\nu$ (pyr-half-ring) \\
\hline$\nu_{6}$ & $A_{\mathrm{g}}$ & $953 / 946$ & $957 / 951$ & $-4 /-5$ & $\nu$ (pyr-breathing) \\
\hline$\nu_{24}$ & $B_{\lg }$ & $863 / 787$ & $859 / 795$ & $4 /-8$ & $\delta$ (pyr-deform) \\
\hline$\nu_{32}$ & $B_{\lg }$ & $816 / 773$ & $823 / 782$ & $-7 /-9$ & $\delta$ (pyr-deform) \\
\hline$\nu_{16}$ & $A_{\mathrm{g}}$ & 727 & 733 & -6 & $\delta($ pyr-deform $)$ \\
\hline$\nu_{7}$ & $A_{\mathrm{g}}$ & 717 & 723 & -6 & $\delta($ pyr-deform $)$ \\
\hline$\nu_{33}$ & $B_{\lg }$ & 416 & & & $\delta$ (pyr-rot.) \\
\hline$\nu_{25}$ & $B_{\lg }$ & 401 & 402 & -1 & $\delta$ (pyr-rot.) \\
\hline$\nu_{8}$ & $A_{\mathrm{g}}$ & 299 & 306 & -7 & $\delta($ pyr-trans $)$ \\
\hline$\nu_{18}$ & $A_{\mathrm{g}}$ & 154 & 158 & -4 & $\delta($ pyr-trans $)$ \\
\hline$\nu_{35}$ & $B_{\mathrm{lg}}$ & 94 & 105 & -11 & $\delta($ pyr-trans $)$ \\
\hline
\end{tabular}

athe symmetry under $D_{2 h}$ point group;

${ }^{\mathrm{b}} \Delta=f_{\mathrm{H}_{2} \mathrm{MPP}}($ Cal. $)-f_{\mathrm{H}_{2} \mathrm{MPPP}}\left(\mathrm{Obs}\right.$.); ${ }^{\mathrm{c}}$ according to Ref. [3,13] 
$\mathrm{C}_{\beta} \mathrm{C}_{\beta}$ 键间的相同相位的振动标记为 $\nu_{2}$ 振动, 相反相 位的振动标记为 $\nu_{11}$ 振动. $\nu_{2}$ 带和 $\nu_{11}$ 带的理论计算 频率分别为 1561 和 $1509 \mathrm{~cm}^{-1}$, 分别对应于实测光 谱中的 1544 和 $1492 \mathrm{~cm}^{-1}$.

\subsection{3 吡咯环四分之一振动}

$\nu_{20}$ 简正振动表示质子化吡咯环的四分之一环 振动, 在 $\mathrm{H}_{2} \mathrm{MPP}$ 中, 该振动分裂成质子化吡咯环 $\mathrm{I}$ 和吡咯环 III各自独立的四分之一振动, 相应的计算 振动频率为 $1373 \mathrm{~cm}^{-1}$ 和 $1388 \mathrm{~cm}^{-1}$, 分别对应实测 光谱中的 1378 和 $1387 \mathrm{~cm}^{-1}$ 带, 非质子化吡咯环的 四分之一环振动 $\nu_{29}$ 的计算频率为 $1314 \mathrm{~cm}^{-1}$, 对应 实测光谱中的 $1307 \mathrm{~cm}^{-1}$. 计算没有显示该简正振动 发生分裂.

\subsection{4 吡咯环二分之一环振动}

$\nu_{4}$ 和 $\nu_{12}$ 简正振动分别表示质子化吡咯环和非 质子化吡咯环的对称二分之一环振动. 相应的理论 计算频率分别为 1356 和 $1406 \mathrm{~cm}^{-1}$, 分别对应于实 测的 1354 和 $1406 \mathrm{~cm}^{-1}$ 带. $\nu_{22}$ 和 $\nu_{30}$ 简正振动分别 表示质子化吡咯环和非质子化吡咯环的不对称二分 之一环振动. 这两个振动的计算频率分别位于 974 和 $1002 \mathrm{~cm}^{-1}$. 相应的实测频率为 975 和 $1002 \mathrm{~cm}^{-1}$.

\subsection{5 吡略环呼吸振动}

$\nu_{6}$ 简正振动表示质子化吡咯环和非质子化吡咯 环间相同相位的呼吸振动. 理论计算显示 $\mathrm{H}_{2} \mathrm{MPP}$ 的 $\nu_{6}$ 振动分裂成为两个简正振动: 一个主要包括非质 子吡咯环 $\mathrm{IV}$ 与质子化吡咯环间的相同相位的振动, 其计算和实测频率分别为 946 和 $951 \mathrm{~cm}^{-1}$; 另一个 主要包括非质子吡咯环 II 和质子化吡咯环间的相同 相位振动. 其计算和实测频率分别位 953 和 957 $\mathrm{cm}^{-1} . \nu_{6}$ 简正振动的分裂同样应该归因于单个苯基 团取代导致的同类吡咯大环结构上的差异. 质子化 吡咯环和非质子化吡咯环的相反相位呼吸振动 $\nu_{15}$ 没有发生分裂, 其计算和实测频率分别为 989 和 $992 \mathrm{~cm}^{-1}$.

\subsection{6 吡咯环变形振动}

$\nu_{7}$ 和 $\nu_{16}$ 简正振动都表示对称的吡咯环变形振 动, 相应的计算振动频率为 717 和 $727 \mathrm{~cm}^{-1}$, 分别对 应于实测拉曼谱中的 723 和 $733 \mathrm{~cm}^{-1}$ 带.

$\nu_{24}$ 和 $\nu_{32}$ 简正振动表示两个 $\mathrm{C}_{m} \mathrm{C}_{m}$ 轴的不对称 伸缩振动导致的吡咯环的不对称变形振动, 理论计 算显示, $\mathrm{H}_{2} \mathrm{MPP}$ 的这两个简正振动都发生分裂. $\nu_{24}$ 振动分裂为苯基连接的 $\mathrm{C}_{m}$ 原子相对于卟啉环中心 的往复振动, 和与该 $\mathrm{C}_{m}$ 原子对称的、联接 $\mathrm{H}$ 原子的
$\mathrm{C}_{m}$ 原子相对于卟啉环中心的往复振动. 这两个振动 的计算频率分别为 863 和 $787 \mathrm{~cm}^{-1}$, 分别对应于实 测拉曼光谱中的 859 和 $795 \mathrm{~cm}^{-1}$. 前一振动的振动 结构与 $\mathrm{H}_{2} \mathrm{TPP}$ 的相应振动类似, 这导致二者的振动 频率也相互接近 ${ }^{[4]}$. 同样原因, 后一振动的频率接近 $\mathrm{H}_{2} \mathrm{P}$ 的相应振动. 计算表明 $\nu_{32}$ 简正振动也分裂为联 接苯基的 $\mathrm{C}_{m}$ 原子的振动, 和与之对称的 $\mathrm{C}_{m}$ 原子的 振动. 这两个振动的计算频率分别为 816 和 773 $\mathrm{cm}^{-1}$, 实测的拉曼频率为 823 和 $782 \mathrm{~cm}^{-1}$, 这两个振 动也分别与 $\mathrm{H}_{2} \mathrm{TPP}$ 和 $\mathrm{H}_{2} \mathrm{P}$ 的相应振动有近似的振 动频率 ${ }^{[14]} . \nu_{24}$ 和 $\nu_{32}$ 简正振动的分裂也应该归因于单 个苯基团取代导致的同类吡咯大环结构上的差异.

\subsection{7 叶啉环呼吸运动}

$\nu_{8}$ 简正振动表示对称的吡咯环平动, 该振动导 致整个卟啉大环的扩展和收缩, 故又称为吓啉环呼 吸振动. 它的实测频率和理论计算频率分别为 306 和 $299 \mathrm{~cm}^{-1}$. 值得注意的是, 在实测光谱中 $\nu_{8}$ 带附近 还观察到一较强的 $315 \mathrm{~cm}^{-1}$ 带. 从拉曼振动强度上 来看, 该带可能对应的计算频率为 $311 \mathrm{~cm}^{-1}$, 该振动 在 $\mathrm{H}_{2} \mathrm{P}$ 等中心对称的吓啉分子中为非拉曼活性振 动, 在 $\mathrm{H}_{2} \mathrm{MPP}$ 中, 由于中心对称性消失, 该振动表现 出一定的拉曼强度.

$\nu_{18}$ 简正振动表示不对称的吡咯环平动运动, 该 运动导致卟啉环分别沿 N-N 轴和 NH-HN 轴作相反 相位的伸缩振动. 它的实测频率和理论计算频率分 别为 158 和 $154 \mathrm{~cm}^{-1}$.

\section{3 结 论}

利用 DFT 方法研究了 $\mathrm{H}_{2} \mathrm{MPP}$ 的几何结构和拉 曼光谱. 计算表明单个次甲基桥位上的苯基取代导 致卟啉分子(包括吓啉大环)对称性的降低, 使吓啉 大环结构由正四方形变为类似于菱形的结构; 同时, 单苯基取代导致的卟啉大环的面外形变相对较小. DFT 计算给出的拉曼振动频率(校正因子为 0.971) 与实测频率数据较为吻合 $\left(\mathrm{RMS} \leqslant 6.7 \mathrm{~cm}^{-1}\right)$. 计算表 明, 单苯基取代导致 $\mathrm{H}_{2} \mathrm{MPP}$ 分子的一些拉曼振动, 如 $\nu_{6} 、 \nu_{20} 、 \nu_{24}$ 和 $\nu_{32}$ 等简正振动发生分裂, 理论分析 将此归因于单个苯基团取代导致的各个吡咯环以及 各个次甲基桥位置结构的不均一性.

\section{References}

1 Chen, D. M.; He, T. J.; Cong, D. F.; Zhang, Y. H.; Liu, F. C.

J. Phys. Chem. A, 2001, 105: 3981 
2 Guo, H.; Jiang, J.; Shi, Y.; Wang, Y.; Liu, J.; Dong, S. J. Phys. Chem. B, 2004, 108: 10185

3 Li, X. Y.; Czernuszewicz, R. S.; Kincaid, J. R.; Su, Y. O.; Spiro, T. G. J. Phys. Chem., 1990, 94: 31

4 Bell, S. E. J.; Al-Obaidi, A. H. R.; Hegarty, M. J. N.; McGarvey, J. J.; Hester, R. E. J. Phys. Chem., 1995, 99: 3959

5 Tazi, M.; Lagant, P.; Vergoten, G. J. Phys. Chem. A, 2000, 104: 618

6 Kozlowski, P. M.; Jarzecki, A. A.; Pulay, P.; Li, X. Y.; Zglerski, M. Z. J. Phys. Chem., 1996, 100: 13985

7 Oakes, R. E.; Bell, S. E. J. J. Phys. Chem. A, 2003,107: 10953

8 Chen, D. M.; Liu, X.; He, T. J.; Liu, F. C. Chem. Phys. Lett., 2002,
361: 106

9 Wojaczynski, J.; Latos-Grazynski, L.; Chmielewski, P. J.; calcar, P. V.; Balch, A. L. Inorg. Chem., 1999, 38: 3040

10 Frisch, M. J.; Trucks, G. W.; Schlegel, H. B. et al. Gaussian suite Gaussian 03, Revision C.01. Wallingford CT: Gaussian Inc., 2004

11 Becke, A. D. J. Chem. Phys., 1993, 98: 5648

12 Rassolov, V. A.; Pople, J. A.; Ratner, M. A.; Windus, T. L. J. Chem. Phys., 1998, 109: 1223

13 Li, X. Y.; Zgierski, M. Z. J. Phys. Chem., 1991, 95: 4268

14 Zhang, Y. H.; Li, Z. Y.; Wu, Y.; Zhu, Y. Z.; Zheng, J. Y. Spectrochim. Acta A, In press

\title{
DFT Study on the Geometric Structure and Raman Spectrum of meso-monophenylporphyrin $\left(\mathrm{H}_{2} \mathrm{MPP}\right)^{*}$
}

\author{
ZHANG, Ying-Hui RUAN, Wen-Juan WU, Yang \\ (Department of Chemistry, Nankai University, Tianjin 300071)
}

\begin{abstract}
The geometric structure and Raman frequencies of meso-monophenylporphyrin $\left(\mathrm{H}_{2} \mathrm{MPP}\right)$ were studied by density-functional-theory(DFT). The calculation indicates that meso-monophenyl substitution induces larger distortion to meso-position than to pyrrole ring. All calculated frequencies were scaled with a single factor of 0.971, which gives acceptable results compared with experimental data (the RMS is $6.7 \mathrm{~cm}^{-1}$ ). The measured Raman spectra were discussed and the assignment of in-plane vibrational normal mode of porphyrin ring was given on the basis of theoretical calculation. Further analysis indicates the splits of some in-plane vibrational normal modes of porphyrin ring (for instance $\nu_{6}, \nu_{20}, \nu_{24}$, and $\nu_{32}$ mode), which was attributed to the diversification of the structure around $\mathrm{C}_{m}$ atoms and pyrrole structure induced by meso-monophenyl substitution.
\end{abstract}

Keywords: DFT, meso-monophenylporphyrin, Raman spectrum

Received:May 16,2005; Revised:July 29, 2005. * The Project Supported by NSFC(20303009, 20271030)
Correspondent:ZHANG, Ying-Hui(E-mail: zhangyhi@nankai.edu.cn; Tel:022-81177012). 